\title{
Consequences of Heavy Quark Symmetries for Hadronic Molecules
}

\author{
Carlos Hidalgo-Duque* \\ Instituto de Física Corpuscular (IFIC), Centro Mixto CSIC-Universidad de Valencia, Institutos \\ de Investigación de Paterna, Aptd. 22085, E-46071 Valencia, Spain
}

\section{Feng-Kun Guo}

Helmholtz-Institut für Strahlen- und Kernphysik and Bethe Center for Theoretical Physics, Universität Bonn, D-53115 Bonn, Germany

\section{Juan Nieves}

Instituto de Física Corpuscular (IFIC), Centro Mixto CSIC-Universidad de Valencia, Institutos de Investigación de Paterna, Aptd. 22085, E-46071 Valencia, Spain

\section{Pavón Valderrama}

Institut de Physique Nucléaire, Université Paris-Sud, IN2P3/CNRS, F-91406 Orsay Cedex, France

In this work, an analysis under the assumption that the $X(3872)$ and the $Z_{b}(10610) / Z_{b}^{\prime}(10650)$ are heavy meson-heavy antimeson molecular states is presented. It is based on an Effective Field Theory (EFT) approach built thanks to the high degree of symmetry that these molecular states have. For that purpose, the most general leading order lagrangian, compatible with the symmetries, is found, and its Low Energy Constants (LECs) fitted to experimental data. Next, we solve the Lippmann-Schwinger Equation (LSE) for different channels finding several molecular partners of the resonances assumed to have a molecular structure. Finally, considering the Heavy Antiquark-Diquark Symmetry (HADS) we are able to extend the previous meson analysis to a baryon sector where exotic pentaquark-like states are found.

XV International Conference on Hadron Spectroscopy-Hadron 2013

4-8 November 2013

Nara, Japan

\footnotetext{
* Speaker.
} 


\section{Introduction}

The full comprehension of QCD is an important task in particle physics. Numerous problems have been found and, though many breathtaking advances have been made, there are still many unsolved puzzles.

One of these puzzles we are interested is the complete understanding of the full hadronic spectrum of QCD. There are different states, those theorized in simple quark models, such as mesons and baryons; and some quite more exotic, namely, tetraquarks, pentaquarks, glueballs, hadronic molecules, etc. In this work we are focusing in the molecular part of this QCD spectrum. The heavy meson-antimeson molecular states were theorized for the first time in the 70s [1]. This assumption was made based on the existing similarities between these molecular states and the nucleon-nucleon system. In both cases, bound states would be caused by meson exchanges. So, the existence of a nucleon-nucleon bound state, that is the deuteron, would be the main reason to believe that meson-antimeson bound states could also be possible in nature.

This theoretical assumption was quickly tested for different resonances but the clearest candidate to be a meson-antimeson molecule was the popular $X$ (3872), discovered in 2003 [2]. This exotic resonance in the hidden charm sector could not be a charmonium excited state because of its possible quantum numbers, recently confirmed as $J^{P C}=1^{++}[3]$ and it was really close to the $D \bar{D}^{*}$ threshold (low binding energy, as in the deuteron, is an expected characteristic of molecular states.). Since then, many other $X, Y, Z$ states have been reported and we cannot still know how many will fit into this molecular scheme, being the $Z_{b}(10610) / Z_{b}^{\prime}(10650)$ [4] two of the most promising ones.

In this work we aim to advance the current knowledge of the QCD spectrum using an effective lagrangian based on the many symmetries heavy meson-heavy antimeson systems should have. We will assume the molecular nature of a couple of resonances: the $X(3872)$ as a $J^{P C}=1^{++} D \bar{D}^{*}$ state and the isovector $Z_{b}(10610) / Z_{b}^{\prime}(10650)$ as a $J^{P C}=1^{+-} B \bar{B}^{*} / B \bar{B}^{*}$. Hence, under these assumptions we will fit some undetermined Low Energy Constants (LECs) in our effective lagrangian thus, when using that fitted dynamics in different channels we will be able to make our predictions.

\section{Symmetries, Effective Lagrangian, determination of the LECs and LSE}

In this section we briefly explain our analysis. For more details, see our previous works $[5,6$, 7]. Our approach to this analysis is based on the symmetries that the heavy meson-heavy antimeson have. The origin of these symmetries can be traced back to different scales that are relevant in these systems: molecular ratio, mesonic ratio, $\Lambda_{Q C D}, M_{Q} \ldots$ We can distinguish two different kinds of symmetries depending on the quarks involved: symmetries due to the light quarks of the system and symmetries related to the heavy quarks.

Symmetries due to the light quarks are $S U(3)$ light quark flavour symmetry, which can relate this analysis for different isospin states since the $D_{(s)}^{(*)}\left(\bar{D}_{(s)}^{(*)}\right)$ mesons (antimesons) will be described into triplets and chiral symmetry, which will constrain pion exchanges.

The symmetries due to the presence of a heavy quark we are using in this work are:

- Heavy Quark Spin Symmetry (HQSS), which states that the dynamics is independent of the heavy quark spin. This will have two important consequences: the effective lagrangian will be quite simplified and heavy molecules will be arranged in HQSS multiplets. 
- Heavy Quark Flavour Symmetry (HFS), which states the independence on the heavy quark flavour. Hence, charm and bottom sector should be similar.

- Heavy Antiquark Diquark Symmetry (HADS), which relates a heavy diquark with a heavy antiquark.

At leading order, the most general effective potential that respects HQSS is [8]:

$$
\begin{aligned}
V_{4}= & +\frac{C_{A}}{4} \operatorname{Tr}\left[\bar{H}_{a}^{(Q)} H_{a}^{(Q)} \gamma_{\mu}\right] \operatorname{Tr}\left[H_{a}^{(\bar{Q})} \bar{H}_{a}^{(\bar{Q})} \gamma^{\mu}\right]+\frac{C_{A}^{\lambda}}{4} \operatorname{Tr}\left[\bar{H}_{a}^{(Q)} \lambda_{a b}^{i} H_{b}^{(Q)} \gamma_{\mu}\right] \operatorname{Tr}\left[H_{c}^{(\bar{Q})} \lambda_{c d}^{i} \bar{H}_{d}^{(\bar{Q})} \gamma^{\mu}\right]+(2.1) \\
& +\frac{C_{B}}{4} \operatorname{Tr}\left[\bar{H}_{a}^{(Q)} H_{a}^{(Q)} \gamma_{\mu} \gamma_{5}\right] \operatorname{Tr}\left[H_{a}^{(\bar{Q})} \bar{H}_{a}^{(\bar{Q})} \gamma^{\mu} \gamma_{5}\right]+\frac{C_{B}^{\lambda}}{4} \operatorname{Tr}\left[\bar{H}_{a}^{(Q)} \lambda_{a b}^{j} H_{b}^{(Q)} \gamma_{\mu} \gamma_{5}\right] \operatorname{Tr}\left[H_{c}^{(\bar{Q})} \lambda_{c d}^{j} \bar{H}_{d}^{(\bar{Q})} \gamma^{\mu} \gamma_{5}\right]
\end{aligned}
$$

where the $C^{\prime}$ s are four free undetermined LECs, $H(\bar{H})$ is a meson (antimeson) hyperfield that contains both the pseudoscalar and vector mesons, and the $\lambda$ 's are the Gell-Mann matrices, as we assumed $S U$ (3) light quark flavour symmetry (they would be the Pauli matrices, in case only $S U$ (2) was considered). The four LECs can be rewritten through a linear combination into $C_{0 A}, C_{0 B}, C_{1 A}$ and $C_{1 B}$, where $I=0,1$ is refering to the isospin channel where this counterterm is needed.

Then, in order to determine the LECs in the lagrangian, we make use of the assumptions specified in Section1. Under these two assumptions we can fix the following linear combinations of the LECs:

$$
X(3872) \Rightarrow\left\{\begin{array}{l}
C_{0 X}=C_{0 A}+C_{0 B} \\
C_{1 X}=C_{1 A}+C_{1 B}
\end{array} \quad Z_{b}(10610) \Rightarrow C_{1 Z}=C_{1 A}-C_{1 B}\right.
$$

Two linear combinations of LECs arise from the $X$ (3872) assumption because, we are including in the analysis not only the mass of the resonance but its main decay channels too. So far, it is a puzzle the reason why the main two decays of the $X(3872)$ appear in different isospin channels according to the experimental ratio of branching franctions [9]:

$$
\frac{\mathscr{B}(X(3872) \rightarrow J / \psi \overbrace{\pi^{+} \pi^{-} \pi^{0}}^{\omega}}{\mathscr{B}(X(3872) \rightarrow J / \psi \underbrace{\pi^{+} \pi^{-}}_{\rho})} \simeq 0.8 \pm 0.3 . \quad \Rightarrow \quad R_{X}=\frac{\mathscr{M}(X \rightarrow J / \psi \rho)}{\mathscr{M}(X \rightarrow J / \psi \omega)}=0.26_{-0.05}^{+0.08}
$$

where this experimental ratio have been translated to a ratio of decay amplitudes $R_{X}$, just taking into account the different decay widths of the intermediate $\rho$ and $\omega$. These two ratios should be zero or infinite if the $X(3872)$ had well defined isospin and isospin were conserved in the process. To explain these finite ratios there are two different scenarios: either the $X(3872)$ isospin is well defined and isospin is not conserved in its decays or isospin is a conserved quantity in the decay but the $X(3872)$ isospin is not well defined. We will work in this latter scenario. Though isospin is a good quantum number in the effective lagrangian, it is not in the kinetic term of the whole hamiltonian. The mass difference between the neutral component $\left(D^{0} \bar{D}^{0}\right)$ and the charged component $\left(D^{+} D^{-}\right)$is around $8 \mathrm{MeV}$. However, since the mass of the $X(3872)$ resonance is so close to the $D \bar{D}^{*}$ this small mass difference is not negligible and isospin turns out not to be a good quantum number. 
Therefore, we will work with a two coupled channels potential with a non-vanishing $I=1$ interaction that we will fit to reproduce the value of the ratio $R_{X}$ in Eq. 2.3.

Next, we solve the standard LSE in the different channels we are considering, introducing a Gaussian regulator to treat the ultraviolet divergences in the following way:

$$
\begin{gathered}
T=V+V G T \\
\left\langle\vec{p}|T| \vec{p}^{\prime}\right\rangle=\left\langle\vec{p}|V| \vec{p}^{\prime}\right\rangle+\int d^{3} \vec{k} \frac{\langle\vec{p}|V| \vec{k}\rangle\left\langle\vec{k}|T| \vec{p}^{\prime}\right\rangle}{E-m_{1}-m_{2}-\frac{k^{2}}{2 \mu}} \\
\left\langle\vec{p}|V| \vec{p}^{\prime}\right\rangle=V\left(\vec{p}, \vec{p}^{\prime}\right)=v e^{-\vec{p}^{2} / \Lambda^{2}} e^{-\vec{p}^{\prime 2} / \Lambda^{2}} \quad \Rightarrow \quad G=\int \frac{d^{3} \vec{k}}{(2 \pi)^{3}} \frac{e^{-2 \vec{k}^{2} / \Lambda^{2}}}{E-m_{1}-m_{2}-\frac{k^{2}}{2 \mu}}
\end{gathered}
$$

\section{Results in the meson sector}

Within the scheme defined up to now, we have solved the LSE for several channels (those whose dynamics does not depend on the $C_{0 A}$ LEC). The resonances we find and predict as heavy meson-heavy antimeson molecular partners of the $X(3872)$ and $Z_{b}(10610) / Z_{b}^{\prime}(10650)$ are shown in Table II in [10]. In this table we can see the mesonic molecular partners of the $X(3872)$ and the $Z_{b}(10610) / Z_{b}^{\prime}(10650)$. The input in this analysis has been the $X(3872)$ mass and decay amplitude ratio and the $Z_{b}(10610)$ mass. First thing we can notice is that $Z_{b}^{\prime}(10650)$ appears naturally in the isovector sector as a consequence of these assumptions, as one would have expected. Besides, there is also an important prediction in the $J^{P C}=2^{++} D^{*} \bar{D}^{*}$ channel. There, we predict the existence of a resonance since this state has the same dynamics and a similar reduced mass than the $X(3872)$ resonance. Its existence would be a strong hint for the molecular nature of the $X(3872)$.

In the isovector sector, this analysis leads two important consequences too. In this model we obtain a couple of resonances as virtual states with $J^{P C}=1^{+-}$and structure $D \bar{D}^{*}$ and $D^{*} \bar{D}^{*}$, respectively. These two resonances can be interpreted as the recently discovered $Z_{c}(3900)$ [11, 12] and $Z_{c}^{\prime}(4025)$ [13], and we are able to assign to them quantum numbers $\left(J^{P C}=1^{+-}\right)$, still experimentally undetermined.

\section{Results in the baryonic sector}

So far, our analysis has only taken advantage of HQSS (which greatly simplified the effective lagrangian) and HFS (which related results in the charm with the bottom sector, and viceversa). But there was another extra heavy quark symmetry we have not used yet: HADS. This symmetry, first theorized in the 90s [14] states that a heavy diquark behaves as a heavy antiquark up to corrections of the order $\left(\frac{1}{m_{Q^{v}}}\right)$, being $0<v<1$ the velocity of the heavy diquark system. These corrections are a little bit higher than those arising in HQSS and HFS which are of order $\left(\frac{1}{m_{Q}}\right)$.

If a heavy diquark behaves as a heavy antiquark we can now use our previous analysis in the meson sector to describe heavy meson-doubly heavy baryons since the dynamics only depends on the light degrees of freedom which are the same in both systems. For the same reason, the previous fits for the different LECs are still valid and can also be used in this context. 
In this way we obtain the results shown in Table II of Ref. [15], where our baryon predictions can be found. As this is a very qualitative analysis, there are not many strong conclusion that can be drawn from it, besides that resonances in the scalar sector seem to be deeper bound than those in the vector sector.

\section{Conclusions}

In this work, we have analysed heavy meson-heavy antimeson systems taking advantage of an effective lagrangian based on the heavy quark symmetries these molecular states are supposed to have. We have fitted the undetermined parameters of the lagrangian to reproduce experimental data: the $X(3872)$ and the $Z_{b}^{\prime}$ s. The validity of our analysis strongly depends on the goodness of these assumptions. In this way we have been able to predict a whole set of molecular partners both in the meson and baryon sector.

It is worth to notice that we have been working at leading order and, consequently, coupled channel and pion exchange effects have not been considered as they are subleading [5, 6].

\section{Acknowledgments}

C. H.-D. thanks the support of the JAE-CSIC Program. This work is supported in part by the DFG and the NSFC through funds provided to the Sino-German CRC 110 "Symmetries and the Emergence of Structure in QCD”, by the NSFC (Grant No. 11165005), by the Spanish Ministerio de Economía y Competitividad and European FEDER funds under the contract FIS2011-28853C02-02 and the Spanish Consolider-Ingenio 2010 Programme CPAN (CSD2007-00042), by Generalitat Valenciana under contract PROMETEO/2009/0090 and by the EU HadronPhysics2 project, grant agreement no. 227431.

\section{References}

[1] M. B. Voloshin and L. B. Okun, JETP Lett. 23 (1976) 333 [Pisma Zh. Eksp. Teor. Fiz. 23 (1976) 369].

[2] S. K. Choi et al. [Belle Collaboration], Phys. Rev. Lett. 91 (2003) 262001.

[3] RAaij et al. [LHCb Collaboration], Phys. Rev. Lett. 110 (2013) 222001

[4] A. Bondar et al. [Belle Collaboration], Phys. Rev. Lett. 108 (2012) 122001.

[5] M. P. Valderrama, Phys. Rev. D 85 (2012) 114037.

[6] J. Nieves and M. P. Valderrama, Phys. Rev. D 86 (2012) 056004.

[7] C. Hidalgo-Duque, J. Nieves and M. P. Valderrama, Phys. Rev. D 87 (2013) 076006.

[8] M. T. AlFiky, F. Gabbiani and A. A. Petrov, Phys. Lett. B 640 (2006) 238.

[9] C. Hanhart, Y. .S. Kalashnikova, A. E. Kudryavtsev and A. V. Nefediev, Phys. Rev. D 85 (2012) 011501.

[10] F. -K. Guo, C. Hidalgo-Duque, J. Nieves and M. P. Valderrama, Phys. Rev. D 88 (2013) 054007.

[11] M. Ablikim et al. [BESIII Collaboration], Phys. Rev. Lett. 110 (2013) 252001.

[12] Z. Q. Liu et al. [Belle Collaboration], Phys. Rev. Lett. 110 (2013) 252002.

[13] M. Ablikim et al. [BESIII Collaboration], arXiv:1308.2760 [hep-ex].

[14] M. J. Savage and M. B. Wise, Phys. Lett. B 248 (1990) 177.

[15] F. -K. Guo, C. Hidalgo-Duque, J. Nieves and M. P. Valderrama, Phys. Rev. D 88 (2013) 054014. 